et elle sera réalisée en Allemagne cette année. Le flacon normal, dans des caisses normales: voilà ce qui facilite également le travail dans la laiterie. L'exploitation dans tous les services est améliorée, ainsi que le mode de distribution et connaissant l'encombrement des caisses, il devient possible d'organiser comme il faut leur convoiement dans la laiterie.

Les voitures qui font la livraison directe au consommateur doivent être construites de façon qu'on puisse y mettre 400 litres de lait en bouteilles, tandis que les voitures qui font la livraison en gros et qui sont bien isolées doivent pouvoir contenir de 1.000 à 3.000 litres.

La distribution du lait en bouteilles nous donne également la possibilité de diminuer les frais des succursales dans les grandes villes. On peut en effet, entrevoir la possibilité de diminuer le nombre de chambres frigorifiques et, avec elles, le travail de déchargement qui s'y trouve intimement lié. A notre avis, le lait en bouteilles serait laissé le soir dans les grandes automobiles isolées logées dans les cours des succursales pour être distribué le lendemain directement aux voitures de détail.

Tous les progrès réalisés dans ces dernières années dans le transport du lait, à quelque temps que ce soit, sont déjà grands et, à n'en pas douter, il faciliteront, par leur développement, l'exploitation de la laiterie dans les grandes villes, en même temps qu'ils permettront aux consommateurs de recevoir un lait à boire encore meilleur.

\title{
CONTRIBUTION A L'ÉTUDE DU LAIT STÉRILISÉ
}

\author{
par M. PELLET, \\ Ingénieur agronome.
}

La stéritisation du lait a été jusqu'ici pratiquée avec un empirisme incompatible avec la constance des résultats. Les méthodes très variées s'inspirent davantage de préférences personnelles que de règles scientifiquement établies. La stérilisation absolue ne peut être obtenue sans altérer les qual tés physiques et biologiques du lait; elle ne doit avoir d'autre objectif que la sécurité et la conservation dans des limites normales d'emploi. Les autoclaves sont d'un maniement plus délicat qu'on ne se le figure. Echauffement de l'atmosphère intérieure, relations avec la pression, distribution de la vapeur, température au sein des liquides traités, durée effective de la stérilisation, sont autant de points dont on s'est peu inquiété. Sollicité par l'étude de ces divers états du problème, j'avais exposé au Congrès International de Laiterie (Mai 1926) une technique issue de mes observations à la Laiterie municipale de Nantes.

Simultanément, M. Pellet donna t dans le "Lait" (Septembre 1926, $\left.N^{\circ} 58\right)$ un important travail sur la Marche de la température dans un stérilisateur industriel. 
Il nous apporte aujourd'hui le complément de recherches poursuivies avec autant de soin que de précision, et où l'ingéniosité ne le cède en rien à la patience de l'expérimentateur. Le travail de $M$. Pellet qui a obtenu l'honneur d'une récompense de l'Association des anciens élèves de l'Institut agronomique, est la première étuded'ensemble sur la techniquede la stérilisation du lait.

Elle se recommande hautement aux spécialistes pour qui elle sera un guide précieux, et aussi à ceux qui, utilisant des autoclaves, voudront renoncer à un empirisme hasardeux, au profit des méthodes scientifiques garantissant la stabilité des fabrications.

\section{Dr RoBIN,}

Directeur du Bureau d'Hygiène de Nantes. $*^{*} *$

Si l'on consulte, dans les différents livres ou dans les diverses revues scientifiques qui traitent des questions laitières, les documents relatifs à la préparation du lait stérilisé, on trouve sur ce sujet des renseignements assez nombreux mais qui ne semblent pas toujours concorder entre eux : les exposés des méthodes de stérilisation ne sont pas toujours très précis ; s'il s'agit par exemple de formules de chauffe, les températures maxima différeront ainsi que les temps de séjour à ces températures sans d'ailleurs qu'il apparaisse une corrélation bien nette entre les unes et les autres.

A vrai dire, les auteurs de ces articles, dans lesquels nous avons puisé d'ailleurs d'excellentes choses, sont peut-être dans le vrai en demeurant dans une certaine réserve, car le problème est évidemment assez complexe; nous ne songerons donc pas nous-même à vouloir le résoudre d'une façon définitive, mais tenterons simplement d'apporter à la question un peu de précision, d'une part en coordonnant ce qui a déjà été publié, d'autre part en nous aidant de quelques-uns de nos essais.

Mais avant d'entreprendre la question de la préparation du lait stérilisé, il nous paraît nécessaire de nous livrer tout d'abord à une étude rapide des principaux éléments du lait; puis nous étudierons les agents d'altération du lait. Après le mal, les remèdes : nous examinerons donc ensuite les différents agents de conservation et leur action tant sur les éléments du lait que sur les agents d'altération.

Nous passerons ultérieurement en revue les modes de conservation préconisés par différents auteurs et finalement, après avoir donné les résultats de nos essais sur quelques-unes de ces méthodes, nous exposerons les conclusions que nous avons cru devoir tirer de cette étude.

Disons tout de suite que, malheureusement, notre travail n'est pas aussi complet que nous l'aurions désiré : certaines méthodes de stérilisation nécessitent en effet des appareils tout à fait spéciaux et nous n'avons 
pu, de ce fait, les essayer personnellement. Nous les avons toutefois décrites succinctement en mentionnant les avantages et les inconvénients qui semblent pouvoir résulter de leur application.

\section{I, - LES ÉlÉmientS DU LAIT QU'IL Y A LIEU D'ENVISAger AU POINT DE VUE DE SA CONSERVATION}

Nous employons à dessein le mot de conservation au lieu de stérilisation qui n'est pas assez général ; c'est qu'en effet, il faut envisager les trois types de conservation suivants: physique, chimique et microbiologique.

Par conservation physique, nous entendons que le lait conservé en bouteille devra présenter un aspect semblable au lait normal fraîchement recueilli.

Par conservation chimique, nous entendons que le lait ne devra pas, autant que possible, être altéré par des réactions d'ordre chimique.

Par conservation microbiologique, nous comprenons la stérilisation proprement dite, ou tout au moins l'arrêt du développement microbien

Les éléments du lait qui nous intéressent à ce triple point de vue sont: la matière grasse, le lactose, la caséine, l'albumine, les phosphates, les lécithines, certaines matières minérales, l'acide carbonique, les diastases et les vitamines.

La matière grasse est à considérer surtout au point de vue des conservations physique et chimique. D'un côté en effet, les globules gras tendent à remonter et à constituer un magma crèmeux qu'une agitation ultérieure serait impuissante à émulsionner de nouveau; les secousses subies au cours des transports pourraient même provoquer un barattage partiel de la crème, montée en partie à la surface. Il y aura donc lieu d'éviter cette montée. D'un autre côté, la matière grasse, à la température ordinaire, s'oxyde au contact de l'air et cette oxydation donne lieu à un goût de suif désagréable; il faudra chercher à éviter cette réaction.

Le lactose, l'albumine, la caséine, le phosphocaséinate de chaux, les lécithines, contrairement à la matière grasse, ne sont pas sujets à des modifications physiques ou chimiques à la température ordinaire ; mais ces éléments pourront être plus ou moins modifiés par l'action d'agents de conservation et particulièrement par la chaleur. L'acide phosphorique qui entre dans la composition des trois derniers éléments (1) devra attirer plus spécialement notre attention.

Les principales matières minérales, en dehors des composés que nous venons de voir, sont: le calcium, sous forme de phosphates bicalcique et tricalcique, et de citrate; le sodium (chlorure, phosphate et citrate) ; le potassium (chlorure). Il faut noter également l'acide carbonique qui,

(1) La caséine est une phosphoprotéine et les lécithines sont des dérivés lipo-phosphoriques, 
existant après la traite dans une proportion moyenne de $10 \%$ en volume, tombe en général vers $4 \%$ par suite de l'agitation en cours de transport dans les bidons. Cet acide joue un rôle important dans la solubilisation des sels de chaux. Notons que le citrate de sodium favorise également la solubilisation du phosphate de chaux.

Les diastases du lait ne semblent pas avoir une importance considérable ; ainsi que le dit le Professeur Porcher, "elles varient en quantité et en qualité suivant le lait considéré; elles ne semblent pas dépendantes du mécanisme de la sécrétion et dans ces conditions leur influence est toute problématique; ce sont des "à côté " qui sont loin d'avoir I'importance des diastases digestives ». Le Professeur Hopkins conclut également que "les diastases du lait sont en quantité trop faible pour avoir un effet quelconque, et que leurs propriétés sont telles qu'elles n'ont aucune fonction physiologique dans la nutrition".

Les vitamines ne correspondent pas à des éléments nutritifs du lait; néanmoins elles sont absolument nécessaires soit à la croissance, soit à l'entretien. Dans le cas où le lait sera employé seulement en partie dans l'alimentation humaine, la présence des vitamines n'y sera pas absolument indispensable puisqu'il sera possible d'en absorber sous d'autres formes et il ne sera même pas en général nécessaire de pourvoir spécialement à cette absorption, les différentes espèces de vitamines se trouvant dans les aliments variés qui constituent la ration journalière de l'homme. Dans le cas des enfants qui ne sont nourris qu'avec du lait, il en va autrement et il y a lieu de tenir compte de l'importance de ces substances. Il existe plusieurs variétés de vitamines:

Vitamine $A$. - Soluble dans les graisses et dont la carence chez les jeunes animaux provoque un arrêt de croissance, puis la mort.

Vitamine antirachitique (1). - Egalement soluble dans les graisses, elle serait néanmoins différente de la précédente et ne se rencontrerait normalement pas dans le lait des animaux; néanmoins, si l'on fait entrer dans l'alimentation de la mère un produit tel que l'huile de foie de morue riche en facteur antirachitique, le lait sécrété jouira lui-même de propriétés antirachitiques.

Vitamine B. - Soluble dans l'eau, l'alcool, l'éther, le chloroforme ; son absence cause des phénomènes identiques, mais sous la forme connue des symptômes du béri-béri, d'où son nom de vitamine anti-béribérique.

Vitamine C. - Soluble dans l'eau et l'alcool; son absence peut provoquer le scorhut chez les enfants (maladie de Barlow). La teneur du lait en vitamine $\mathrm{C}$ est assez variable, et d'après Seflumann le lait d'été en contient notablement plus que le lait d'hiver (2).

(1) Appelée aussi " vitamine $\mathrm{D}$ ".

(2) Dans la Revue a Lə Lait” de Décembre 1926, Lesné é Vagliano ont traité cette question des vitamines du lait d'une iaçon particulièrement intéressante, et nous y avons puisé largement. 


\section{II. - LES AGENTS D'ALTERATION DU LAIT.}

Ces agents sont de natures tout à fait différentes ; dans l'ordre d'importance ce sont: les microbes, la chaleur, l'oxygène (et par suite l'air) 1a lumière et certains métaux.

A. Les microbes. - Avec les méthodes actuelles de récolte du lait, récolte qui n'est pas, et ne sera probablement pas d'ici fort longtemps, effectuée dans des conditions parfaites d'asepsie, on constate toujours dans ce produit l'existence de différentes espèces microbiennes, et c'est surtout à cause de leur présence et de leur développement (particulièrement favorisé par la composition du lait qui se trouve être un bouillon de culture excellent), de leur action dégradante sur les différents éléments de ce milieu et de leur nocivité possible, qu'il y a lieu d'annihiler leur effets.

En dehors des microbes, un apport même faible de matières diverses qu'on retrouve dans les étables n'est nuilement recommandable et nous n'insisterons pas sur ce point. II va de soi que moins les germes seront nombreux, plus facile sera leur destruction; moins nous introduirons de formes sporulées, moins énergiquement pourra être poussée l'action des agents de conservation. S'il est pratiquement impossible de récolter et de mettre en bouteille un lait de façon aseptique, il est évident que non seulement on peut, mais on doit, réduire au minimum toutes pollutions et toutes contaminations microbiennes.

La flore microbienne peut se diviser en deux catégories : la flore sans spores et la flore avec spores. La destruction des microbes de la première catégorie est assez facile; dans la seconde, certaines spores sont extrêmement résistantes à la chaleur, surtout celles des groupes subtilis et mésentéricus.

Cette résistance est d'ailleurs très variable pour un même groupe.

B. - La chaleur. - Une température comprise entre $15^{\circ}$ et $40^{\circ}-50^{\circ}$ favorise plus ou moins le développement des microbes ; d'autre part une température élevée, si elle est capable de détruire ces derniers, peut provoquer des altérations sur les éléments du lait. Comme la chaleur, appliquée dans certaines conditons, constitue également un agent de conservation, nous étudierons en même temps dans le chapitre IV les effets utiles et nuisibles qu'entraîne son emploi.

C. - L'oxygène de I'air. - L'oxygène peut provoquer à la pression atmosphérique des réactions plus ou moins rapides, soit au cours des manutentions (action destructive de la vitamine $\mathrm{C}$ pendant l'homogénéisation), soit après préparation (oxydation de la matière grasse dans les flacons où le vide n'a pas été fait - oxydation des vitamines).

Un autre inconvénient de la présence de l'air est tiré de la remarque suivante : quand on chauffe un flacon bouché présentant une chambre d'air au-dessus du liquide, la température du gaz reste inférieure à celle. 
du liquide, et la différence peut atteindre plusieurs degrés suivant le pourcentage d'air existant et la rapidité du chauffage. Il se peut donc que, avec l'emploi de bouchons en liège (corps très mauvais conducteur), des particules de lait, projetées sur la face inférieure du bouchon, n'atteignent pas, au cours de la stérilisation, la même température que celle du liquide. Ces particules pourront ne pas être stérilisées et contaminer ultérieurement le lait. Avec le vide, la température sera à peu près uniforme dans toutes les parties internes du flacon.

D. - La Iumière. - Elle facilite l'oxydation lente de la matière grasse et l'action destructive de l'oxygène sur la vitamine C.

E. - Métaux. - La rouille facilite l'oxydation de la matière grasse. D'après les travaux de Hess et Weinstok, Rice et Miscall, Hunziker, etc... la plupart des métaux sont attaqués par le lait par suite de leur solubilité plus ou moins grande dans l'acide lactique et les lactates; il en résulte une saveur désagréable.

Le zine, le fer, l'aluminium sont les plus solubles mais e'est le cuivre et surtout ses alliages qui communiquent la saveur la plus prononcée.

En dehors de la question goût, le cuivre et ses alliages ou sels ainsi que le fer et ses sels, mais à un moindre degré, déterminent, par catalyse, une décomposition partielle du lait généralement accentuée en présence de l'air. L'attaque est d'ailleurs plus forte avec les alliages par suite de l'intervention de phénomènes électrolytiques qui facilitent les réactions. D'autre part, le lait attaque le cuivre moins fortement à l'ébullition que vers $60-65^{\circ}$; il y aura donc lieu de diminuer autant que possible leur temps de contact aux environs de ces dernières températures. Pratiquement l'étain et l'aluminium sont les moins attaqués.

Enfin, même à l'état de traces infimes, la cuivre provoque la destruction de la vitamine $\mathrm{C}$ par catalyse.

Nous pouvons donc noter dès maintenant qu'il y aura lieu en conséquence d'éviter autant que possible le contact du lait avec des métaux autres que l'étain et l'aluminium, et de craindre plus particulièrement celui du cuivre.

\section{III. - LES PROcÉdÉs Et AGENTS dE CONSERVATION.}

A. Conservation physique. - Le seul inconvénient contre lequel nous avons à lutter est la montée de la matière grasse. On y remédie par' l'homogénéisation (GAULIN). Le procédé consiste à diviser par lamellisation du lait sous une pression de 150 à 200 atmosphères et de préférence vers $85^{\circ}$, les globules de matière grasse à un tel degré de finesse que leur force ascensionnelle devient pratiquement nulle. L'homogénéisation s'est généralisée dans toutes les industries de stérilisation du lait. Notons que cette "fixation " des globules gras sera d'autant meilleure que le lait aura été refroidi le plus brusquement possible. 
B. Conservation chimique. - Pour empêcher l'oxydation de la matière grasse et celle de la vitamine $\mathrm{C}$ qui peuvent se produire petit à petit dans les bouteilles, il suffit de recourir au vide, ou à l'emploi d'un gaz inerte au moment du bouchage (azote, acide carbonique). Le vide est d'ailleurs à peu près le seul procédé employé.

C. Conservation microbiologique. - C'est de beaucoup la plus importante. Les agents de conservation microbiologique sont nombreux; examinons-les rapidement:

Premier groupe. - Agents déterminant une élimination partielle du nombre de germes ou un arrêt total ou partiel du développement des microbes, mais sans destruction de ces derniers. Ce groupe comprend : la filtration-centrifuge, la réfrigération, l'oxygène et le vide.

$1^{0}$ Filtration centrifuge. Cette filtration, qui est plutôt une précipitation rapide de certaines matières en suspension (et en particulier de germes microbiens) par l'action de la force centrifuge, s'effectue soit dans des appareils analogues aux écrémeuses tournant à 8,000 tours environ, soit dans des centrifuges à vitesse de rotation moindre (2,000 tours) mais de diamètre un peu plus grand; les premières machines sont les plus employées car pour une même action séparatrice, le temps de passage au filtre s'y trouve considérablement réduit $(7$ secondes au lieu de 10 à 15 minutes, d'après Marshall et Hood).

$2^{\circ}$ Réfrigération. Elle se présente sous deux formes : le simple refroidissement voisin de 5 à $6^{\circ}$, qui ne modifie pas la nature physique du lait, et la congélation qui ne la modifie que temporairement.

Dans les deux cas, il faut avoir recours à des installations frigorifiques non seulement chez le producteur, mais encore au cours des transports, dans les entrepôts, dépôts de vente et chez les clients. C'est évidemment là le principal obstacle à l'emploi du froid.

Le lait simplement refroidi a d'ailleurs l'avantage sur le lait congelé de ne pas nécessiter de reconstitution et la température à maintenir dans les installations frigorifiques est moins élevée.

$3^{\circ}$ Oxygène. L'oxygène a été employé sous pression soit dans des bouteilles épaisses hermétiquement bouchées, soit dans des siphons; son embouteillage est déjà un obstacle à son emploi pratique.

$4^{\circ}$ Vide. Un grand nombre de laits en bouteille sont préparés de façon à présenter un vide parfait; la production du vide est relativement pratique et divers systèmes de bouchage permettent de conserver ce vide pendant une longue période de temps. L'épreuve du coup de marteau constitue de plus une certaine garantie quant à la qualité du bouchage et un signe probable de non-contamination entre l'époque de la préparation et celle de l'emploi.

DeUXiÈme groupe. Agents déterminant une destruction partielle ou totale des microbes. 
Ce groupe comprend : les antiseptiques, l'électricité, les rayons ultraviolets et la chaleur.

$1^{\circ}$ Antiseptiques. Leur emploi est en général interdit par la loi dans tous les pays, sauf l'eau oxygénée dans certaines conditions et l'ozone. le bi-carbonate de soude, le formol, l'acide salicylique, l'acide borique et les borates sont prohibés.

L'eau oxygénée ehimiquement pure est admise à la dose de $2 \mathrm{~cm}^{3}, 5$ par litre sous la condition d'une pasteurisation ultérieure; son emploi ne correspond pas là au but que nous recherchons. L'ozone a été essayé à différentes reprises; nous verrons par la suite qu'il est inemployable.

$2^{\circ}$ Electricité. - On l'a utilisée sous forme de courants alternatifs; pratiquement peu efficace. du moins jusqu'à présent.

$3^{\circ}$ Rayons ultra-violets. Ces rayons qui donnent de bons résultats dans la stérilisation de l'eau ne peuvent être employés pour le lait, étant donné l'épaisseur trop mince sous laquelle le lait devrait être exposé à leur action par suite surtout de la présence de matières colloïdales qui s'opposent particulièrement à leur pénétration.

$4^{\circ}$ Chaleur. C'est l'un des agents les plus commodes à utiliser ; tous les procédés actuerllement en usage ont recours à lui. C'est le véritable agent de stérilisation.

La chaleur est employée en général sur les bouteilles après bouchage, à moins que celui-ci, au moyen de dispositifs variés, n'ait lieu automatiquement dans l'appareil même de stérilisation en fin de chauffage.

On utilise dans ce but des autoclaves à vapeur sous pression ou des bain-marie à liquides dont le point d'ébullition varie avec leur nature ; les autoclaves fixes sont les plus utilisés, mais il existe également des appareils tournants. L'un des inconvénients de l'emploi de la chaleur réside dans la casse des bouteilles. Si cette casse n'est pas prohibitive, elle grève néanmoins les frais de fabrication d'une façon assez sensible, car il y a non seulement perte du récipient mais aussi du lait qu'il contient. Ce pourcentage de casse peut atteindre quelquefois $10 \%$. Les verres à très faible coefficient de dilatation semblent d'un prix encore trop élevé pour entrer dans le domaine industriel, non pas tant à cause de la mise de fonds que leur emploi nécessiterait, mais par suite du prix auquel il faudrait vendre ou consigner le flaconnage à la clientèle; d'autre part, les récipients en aluminium, dont l'utilisation devrait résoudre la question, n'ont pas, auprès des consommateurs, le succès qu'ils devraient avoir, ceux-ci semblant se méfier - à tort du reste à notre avis - des bouteilles en métal. Ces récipients permettent une rapidité de chauffage et de refroidissement que l'on ne peut obtenir avec des flacons en verre ordinaire.

La chaleur peut être également employée avant mise en récipient, en stérilisant les liquides par circulation continue à travers un système chauffant dont il existe plusieurs modèles.

(à suivre) 